PAPER

\section{Some didactical suggestions for a deeper embedding of DC circuits into electromagnetism}

To cite this article: M Cavinato et al 2017 Eur. J. Phys. 38055707

View the article online for updates and enhancements.
Related content

\begin{tabular}{l} 
- $\frac{\text { Surface charges and J H Poynting's }}{\text { disquisitions on energy transfer in }}$ \\
electrical circuits \\
\hline M Matar and R Welti \\
- Charge distributions on DC circuits and \\
Kirchhoff's laws \\
W R Moreau \\
- Potential difference measurements in the \\
presence of a varying magnetic field \\
E Lanzara and R Zangara
\end{tabular}




\title{
Some didactical suggestions for a deeper embedding of DC circuits into electromagnetism
}

\author{
M Cavinato, M Giliberti $i$ and S R Barbieri \\ Dipartimento di Fisica, Universitá degli Studi di Milano, Italy \\ E-mail: marco.giliberti@unimi.it
}

Received 29 March 2017, revised 17 May 2017

Accepted for publication 20 June 2017

Published 21 August 2017

\begin{abstract}
Undergraduate students often encounter great difficulties in understanding Ohm's law and electrical circuits. Considering the widespread students' beliefs and their common mistakes, as they come out from the literature and our teaching experience, we think that a relevant source of these problems comes from the fact that electrical circuits are generally treated separately from the other topics of electromagnetism, with poor reference to the circulation of the electric field. We present here a way to deal with electrical circuits that could help students to overcome their difficulties. In our approach, the electric field is the protagonist and the mathematical tool the students are asked to use is its circulation. In the light of the circulation of the electric field, the experimental Ohm's law is revisited, the concept of electromotive force is discussed and some suggestions to eliminate common misconceptions about the role of a battery in a circuit are presented.
\end{abstract}

Keywords: electrical circuits, Ohm's law, circulation of the electric field, electromotive force

\section{Introduction}

A vast amount of papers in physics education discuss the many difficulties encountered by students in facing electrical circuits (see [1-9], and references therein). These papers (as well as our teaching experience) highlight that even university students enrolled in their first course in electromagnetism believe that the current is used up by a resistor so as to become weaker as it circulates; others think that the battery always provides the same current, independently of the components to which it is connected. Moreover, many students are not 
able to conceptualize an electrical circuit as a whole system and have great difficulties in representing a real lab one in a schematic way: their physical interpretation changes, depending on how physics questions are posed and on how circuits are graphically represented (see also $[10,11]$ ). It is common to meet students who believe that the position of the symbols in a schematic diagram of a single mesh circuit modifies the properties of the circuit itself.

Often, undergraduate students learn the rules to solve simple exercises about circuits, nonetheless without understanding the physical meaning of what they are doing. Even when students are able to manage resistors, potential differences and electric currents, it is as if they were simply solving a riddle. In fact, in most of the standard courses, electrical circuits remain as a detached part of the entire programme of electromagnetism, much more technical than formal and, moreover, not even adequately connected to the laws which rule the behaviour of the electric field.

A written test, containing 15 questions about basic concepts of classical electromagnetism, among which electric and magnetic fields, given to 12 undergraduate students in mathematics who already attended a one semester basic course on electromagnetism (89 hours), revealed that all the students were unable to correctly draw the electric field lines outside a current-carrying wire. Many of them drew the electric field vector orthogonal to the conductor, few of them say that an electric field exists inside the wire and even less realize the presence of an electric field also outside, nor the presence of a magnetic field inside the wire. A similar situation has been evidenced in [5] for what concerns final year high school students.

The foregoing considerations should not surprise since the study of the electrical properties of circuits is generally not clearly related to Maxwell's equations. On the other hand, we have also to point out that the topics of flux and circulation of a vector field, necessary to understand the physical meaning of the Maxwell's equations in their integral form, are typically a hard task for most of the students [5, 12].

Therefore, what we propose here is a didactical path, addressed to undergraduate students, that faces the study of electrical circuits using the mathematical tools of circulation with the help of Maxwell's equations. This attempt of unifying the language and concepts is, in our opinion, a stimulus to better connect the study of electrical circuits to that of classical electromagnetism and, at the same time, to better master the mathematics of the Maxwell's equations as well. An approach somewhat similar to the present one concerning the role played by the electric field, but based on a classical microscopic model which emphasizes not only the role of the field but also that of its sources (surface charge distributions on the wire) can be found in $[6,13,14]$. In our path, instead, we chose to make nearly no reference to microscopic models and to emphasize phenomenological aspects.

\section{The electric field approach to electrical circuits}

\subsection{Ohm's law}

As a first step, we have to develop a consistent description of the electric current flowing in a circuit. We can start from a simple and ready-to-perform experiment: a piece of electric wire, part of an electrical circuit, the ends $A$ and $B$ of which are at a fixed potential difference $\Delta V=V_{A}-V_{B}$, warms up. If $P$ is the power dissipated in that piece of wire, for a small range of temperature, using a calorimeter, we experimentally obtain: 


$$
P=G \Delta V^{2},
$$

where $G$ is a constant that depends on the properties of the wire. Since this warming effect is linked to a potential difference, it seems natural to assume that it is due to some charge in motion under the action of an electric field $\mathbf{E}$ inside the wire.

Denoting with $d q$ the quantity of charge crossing a section of the wire in the time interval $d t$, the corresponding power expended by the electric force is given by:

$$
d P=\frac{d q}{d t} d V
$$

where $d V$ is the potential difference experienced by the charge $d q$. The total power dissipated in the piece of wire between $A$ and $B$ is thus:

$$
P=I \Delta V,
$$

where we have put:

$$
I \equiv \frac{d q}{d t}
$$

with $I$ the so called 'current intensity'.

By equating equation (1) with equation (3) we obtain:

$$
I=G \Delta V \text {. }
$$

From the constancy of $\Delta V$, it follows that $I$ is constant too. Since from the same definition of electric potential, if $V_{A}$ and $V_{B}$ are held fixed, the electric potential of every point of the circuit cannot change in time, no charge density change can occur. These conditions are those of a steady state. Equation (5) is Ohm's law and one can easily realize why the constant $G$ is referred to as 'conductance'. Usually, in most university text-books, current intensity and potential difference are linked through the resistance $R$ of the circuit by the well known relation:

$$
\Delta V=R I, \quad \text { with } R \equiv \frac{1}{G} .
$$

We prefer, on the contrary, to first introduce the concept of conductance, mainly for two reasons: (i) wires, and conductors in general, are objects that do permit current conduction and therefore the Ohm's law is better written in terms of the conductance; (ii) the use of equation (5) instead of equation (6) clarifies that (at least in general) it is the potential difference that is the cause of the current (and not vice versa, as it is often thought by the students [4]).

It is often useful to write equation (5) in terms of local quantities: the electric field $\mathbf{E}$ and the current density $\mathbf{J}$. Let us suppose to have a cylindrical, homogeneous wire of section $S$ and length $L$. From equation (5) we have

$$
\mathbf{J} S=G \mathbf{E} L,
$$

from which we definitively obtain:

$$
\mathbf{J}=\sigma \mathbf{E},
$$

where $\sigma=G L / S$ is a constant called 'conductivity'.

In university basic text-books (as well as in this paper), Ohm's law is introduced with an experimental approach, but, from a didactical point of view, it is important to choose the most suitable approach. We believe that the standard way, which implies the use of voltmeters and amperometers to study the dependence of $I$ on $\Delta V$, is not formative since, if Ohm's law is not yet known, the functioning of these devices cannot be understood, not even in principle. On 


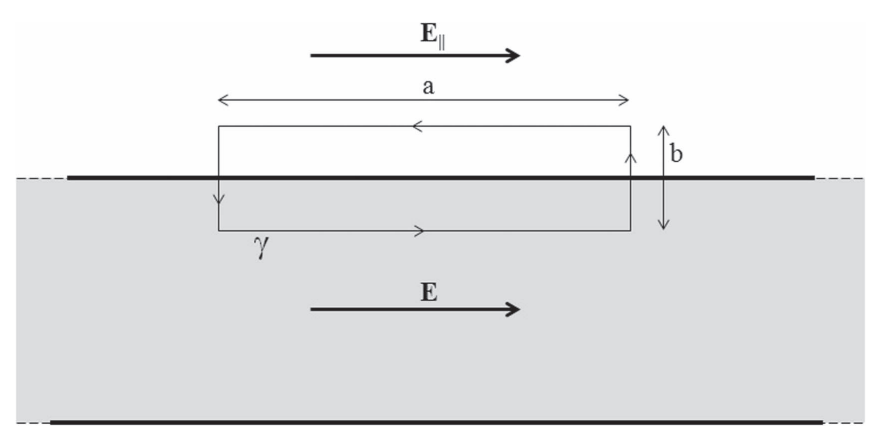

Figure 1. Portion of a current-carrying wire. The circulation of the electric field $\mathbf{E}$ is calculated along the rectangular path $\gamma$.

the contrary, we think that the use of a calorimeter to measure the dissipated power is much more self-consistent.

\subsection{The electric field in an electrical circuit}

As previously said, we are considering a steady state, therefore the electric field is static and conservative. This means that its circulation is zero:

$$
\oint \mathbf{E} \cdot d \mathbf{l}=0
$$

This well known property can dispel many students' misconceptions about the electric field outside a wire that is carrying a steady current. In fact, the majority of students think that the electric field is present only inside the wire. To understand why this opinion is wrong, we can imagine a portion of a current-carrying wire, as in figure 1. We want to show that, just outside the wire, that is at a distance from it much smaller than the wire length, the field $\mathbf{E}$ is not null. For this purpose, let us consider a rectangular path $\gamma$, of sides $a \gg b$, with $b$ much smaller than the wire diameter and placed as in figure 1. Taking into account that the internal field $\mathbf{E}$ is parallel to $\mathbf{J}$, the circulation of $\mathbf{E}$ along $\gamma$ which, according to equation (9) must be zero, implies that an electric field whose component parallel to the wire $\left(\mathbf{E}_{\|}\right)$has intensity and direction equal to the internal field is acting also outside. The qualitative determination of the electric field outside a circuit is certainly very instructive [14, 15] and can be opportunely tackled with students, but, unfortunately, only few papers about it can be found in the literature. In the book by Chabay and Sherwood [13] the topic is clearly discussed and in the related website [16] the electric field can be explored interactively.

\subsection{The circulation of the electrostatic field and the electromotive force}

A source of widespread students' misunderstanding is the concept of the electromotive force (emf) of a battery [17, 18]. Although aware that this problem might distress only European students, our opinion is that much more care should be devoted to discuss this concept. In fact, in many Italian (and also French and Spanish) university text-books we can read that the emf is the circulation of an electric field:

$$
e m f=\oint \mathbf{E} \cdot d \mathbf{l},
$$

where $\mathbf{E}$ is the sum of a conservative field and of a so called 'electromotive field' $\mathbf{E}^{*}$, the circulation of which is the emf. 


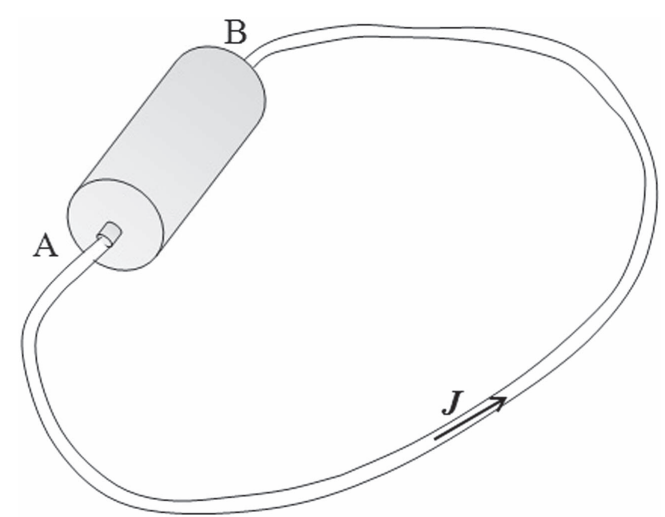

Figure 2. A device (a battery) connected to an electric wire in a simple resistive circuit.

Unless we are in the presence of a time-varying magnetic field, the introduction of this hypothetical field is really questionable, for at least two reasons. First, equation (10) implies that the circulation of $\mathbf{E}^{*}$ is different from zero even in the absence of a time-dependent magnetic field; therefore, $\mathbf{E}^{*}$ cannot obey Maxwell's equations. We struggle to believe it didactically appropriate to call electric a field of this kind, even if its alleged task is to move electric charges. In fact a current flowing in a closed circuit implies the presence of an electric field inside the circuit itself and, therefore, of a device maintaining a potential difference between its poles. The work done by this device on the charges is generally due to a non conservative force, the nature of which depends on the device (which, for example, can be a battery or a Van der Graaf generator, etc). But, except for the case when it comes from a variable magnetic field, it can be very hard, if not impossible, to derive this force from a force field (no one would ascribe to a sort of 'gravitomotive field' the force that a hand exerts on a stone to throw it).

Therefore, in the following we propose a way to define the electromotive force in general, bypassing the concept of electromotive field so as not to conflict with Maxwell's equations and also avoiding the introduction of ill-posed concepts.

Since we are considering a steady situation, the current intensity is constant, the charges' speed does not change and the work $W$ done by the total force $\mathbf{F}$ acting on the charges must be zero. In particular, along the whole circuit:

$$
W=\oint \mathbf{F} \cdot d \mathbf{l}=0
$$

Taking into account all the forces acting on the charge $q$, we can write:

$$
\oint\left(\mathbf{F}_{e l}+\mathbf{F}_{d i s s}+\mathbf{F}_{d e v}\right) \cdot d \mathbf{l}=0
$$

where $\mathbf{F}_{e l}$ is the electrostatic force, $\mathbf{F}_{d i s s}$ is the dissipative force, and $\mathbf{F}_{d e v}$ is the force that the device exerts on the charge $q$. Indicating with $A$ and $B$ the device terminals at potentials $V_{A}$ and $V_{B}$, respectively, with $V_{A}>V_{B}$, equation (12) can also be written as (see figure 2):

$\int_{A}^{B} q \mathbf{E} \cdot d \mathbf{l}+\int_{A}^{B} \mathbf{F}_{d i s s} \cdot d \mathbf{l}+\int_{B}^{A} q \mathbf{E} \cdot d \mathbf{l}+\int_{B}^{A} \mathbf{F}_{d i s s} \cdot d \mathbf{l}+\int_{B}^{A} \mathbf{F}_{d e v} \cdot d \mathbf{l}=0$,

where the direction of the circulation is that of the electric current. In terms of potential difference and work, with obvious notation, we obtain: 


$$
\Delta V_{A B}+\frac{W_{A B}^{d i s s}}{q}-\Delta V_{A B}+\frac{W_{B A}^{\text {diss }}}{q}+\frac{W_{B A}^{d e v}}{q}=0 .
$$

Using the kinetic energy theorem, we get:

$$
\Delta V_{A B}+\frac{W_{A B}^{\text {diss }}}{q}=0,
$$

which implies:

$$
-\Delta V_{A B}+\frac{W_{B A}^{d i s s}}{q}+\frac{W_{B A}^{d e v}}{q}=0 .
$$

If we now define:

$$
e m f \equiv \frac{W_{B A}^{d e v}}{q},
$$

we immediately get:

$$
e m f=\Delta V_{A B}-\frac{W_{B A}^{\text {diss }}}{q},
$$

and we can observe that, since $W_{B A}^{\text {diss }} \leqslant 0, \Delta V_{A B} \leqslant e m f$. Associating the dissipative term $-W_{B A}^{\text {diss }} / q$ with the existence of a device's internal resistance $r$, equation (18) can be written in the usual way:

$$
e m f=\Delta V_{A B}+r I .
$$

Another interesting consideration may be derived from equation (18) when it is written as:

$$
\Delta V_{A B}=e m f+\frac{W_{B A}^{d i s s}}{q} .
$$

Equation (20) clearly indicates that the sum of two quantities that cannot both be expressed as a potential difference does give a potential difference; from this point of view, equation (20) represents the first law of thermodynamics for a system of charge $q$ moving inside the device from $B$ to $A$ :

$$
\Delta U=W+Q .
$$

The energetic balance of equation (14) refers to a closed circuit. If the external conductance is very small, the current intensity flowing in the circuit will be very small too, $\frac{W_{B A}^{d i s s}}{q} \simeq 0$, and, consequently, in the limit of extremely low conductance we can write:

$$
\Delta V_{A B}=e m f,
$$

therefore, from equation (17) we see that $q$, in the limit of very slow charge motion, plays a role similar to that of the absolute temperature $T$ in the definition of the entropy, in the limit of an isothermal, reversible transformation.

In general, one refers to the present situation speaking of an 'open' circuit. We believe that some more attention should be given here because, literally speaking, if the circuit is open $\frac{W_{B A}^{d e v}}{q}=0$ and thus the definition of $e m f$ given by equation (17) would be in contrast with equation (22). Instead, if we think of an open circuit as a circuit with a very small conductance, the problem does not arise. In fact, also from a practical point of view, an experimental determination of the emf of a battery is in general made with a voltmeter, in this way 


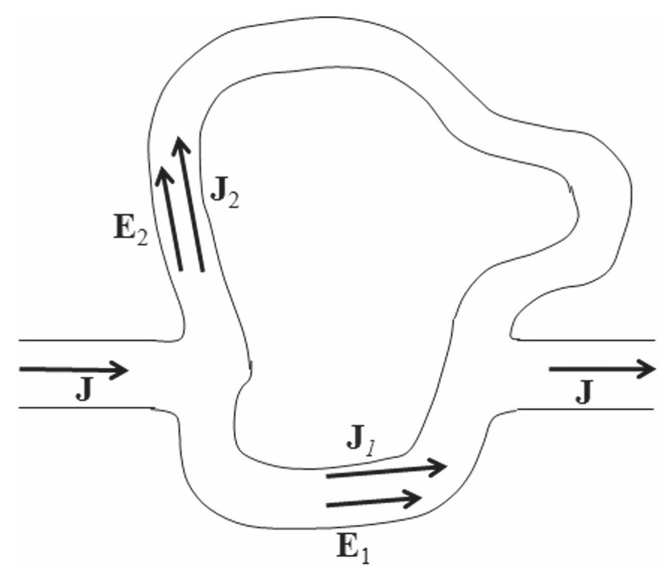

Figure 3. Bifurcation of an electric wire into two parts and their reconnection.

closing the circuit with a low conductance apparatus. We are convinced that a didactical approach that introduces the emf always considering closed circuits could bring students to a greater conceptual understanding of this topic.

Outside the device, using equation (15) we can express the potential difference $\Delta V_{A B}$ as the opposite of the energy dissipated per unit charge between $A$ and $B$ :

$$
\Delta V_{A B}=-\frac{W_{A B}^{d i s s}}{q},
$$

so that, again, $q$ plays a role similar to that of the absolute temperature $T$.

\subsection{Other applications of the electric field circulation}

It is interesting to consider the situation of $N$ resistors in series that can be seen as $N$ different portions of a single mesh circuit that dissipate the energies $W_{1}^{\text {diss }}, W_{2}^{\text {diss }}, \ldots W_{N}^{\text {diss }}$. The sum of the energy losses divided by $q$ must be equal to the potential difference given by equation (15), so that:

$$
\Delta V_{A B}=\Delta V_{1}+\Delta V_{2}+\ldots+\Delta V_{N}
$$

This is the well known Kirchhoff's second law, that is equivalent to the line integral of the electric field between $A$ and $B$ and from which it is immediate to get the equivalent resistance of $N$ resistors in series.

Likewise, let us view a system with $N$ resistors in parallel. Figure 3 shows the bifurcation of a wire into two conducting parts with sections $S_{1}$ and $S_{2}$ which reconnect after the lengths $L_{1}$ and $L_{2}$, respectively. Considering the loop made up of these two parts, calculating the circulation of the electric field along this loop and recalling that $\mathbf{E}$ is parallel to $\mathbf{J}$, with obvious symbology, we get (see also [13]):

$$
E_{1} L_{1}=E_{2} L_{2},
$$

and consequently

$$
R_{1} I_{1}=R_{2} I_{2}
$$

that gives the usual formula for two resistors in parallel. 
A common students' misconception is that a battery is a source of constant current that is used up by the resistors (such as bulbs) [2]. In our experience many instructors tend to correct students by saying that, instead, the battery is a source of constant potential difference. That even this answer is incorrect is evident from equation (19) for the presence of the internal resistance of the device. As an example to clarify this point, let us consider a circuit with a battery of electromotive force $\varepsilon$ and internal resistance $r$ connected to $N$ equal small bulbs in parallel, each of resistance $R$. Then from

$$
\varepsilon=I\left(r+\frac{R}{N}\right)
$$

and from equation (1), the power dissipated by one of the $N$ bulbs results in:

$$
P_{1 ; N}=\frac{\varepsilon^{2} R}{(N r+R)^{2}} .
$$

If the internal resistance is sufficiently small, that is, more precisely, if $N r \ll R$, the power dissipated by a single bulb is nearly the same, both when the bulb is alone and when the circuit contains $N$ identical bulbs in parallel. On the contrary, if the internal resistance is not so small, that is $N r \sim R$, the power dissipated by one bulb starts to significantly decrease with increasing $N$, going to zero like $1 / N^{2}$ for very large $N$. Consequently, in this limit, the total power dissipated by the $N$ bulbs also goes to zero; this would not be the case if the battery were a source of a constant potential difference. The role of internal resistance is crucial for this behaviour and we believe that much more attention should be devoted to it from a didactical point of view.

Up to now we discussed only static situations and, therefore, conservative electric fields. The natural didactical extension is to consider the action of time-dependent fields (for instance in RLC circuits). In the presence of a time-dependent magnetic field, the circulation of the electric field is no longer zero and equation (9) must be modified. With the circuit at rest, at variance with the case of a static electric field, an electromotive field does exist and the electromotive force is given by:

$$
e m f=-\oint \frac{\partial}{\partial t} \mathbf{A} \cdot d \mathbf{l}
$$

where $\mathbf{A}$ is the magnetic vector potential. The physical meaning of the emf of equation (29) is the same as that in the static case with the only difference that, in the present situation, the emf is not localized only in a particular part of the circuit but on every point of it where a timedependent magnetic field is acting. Equation (29) helps us to deeply understand how much the physics of electrical circuits is embedded in the general context of the electromagnetic theory $[19,20]$. The didactics of electric currents induced by a magnetic field is very interesting and will be the subject of a forthcoming paper discussing the important role played by the magnetic vector potential in teaching electromagnetism in secondary school (see also [5]).

\section{Conclusions}

The main problem we tried to overcome with the present work pertains to the too sharp separation, in most university text-books and traditional courses, between electrostatics and electrical circuits. In fact, frequently electrical circuits are but a detached part of the entire programme of electromagnetism, a part neither really technical nor formal, a sort of standalone game. Even when students are able to 'solve' simple circuits, their understanding of the physics involved is often very poor. 
As a way to cure this situation, we made a proposal more grounded on an epistemological point of view than on the evaluation of the students' learning with respect to a traditional approach. In fact, we constructed a much deeper connection between circuits and Maxwell's laws by directly and intensively using the electric field notion and the circulation operator. This gave us also the opportunity to reflect on Ohm's law and to clarify the meaning of electromotive force, an often ill-posed concept. Moreover, the study of resistors in series or in parallel also comes out as a direct application of the circulation of the electric field. The improvement of the epistemological structure of the topic encourages us to suppose that the implementation of this proposal in class might bring students to a better understanding of the subject.

\section{Acknowledgments}

We would like to thank the two referees for their fruitful and highly appreciated comments.

\section{ORCID iDs}

M Giliberti (iD https://orcid.org/0000-0001-5036-3879

\section{References}

[1] Arons A B 1990 A Guide to Introductory Physics Teaching (New York: Wiley)

[2] McDermott L C and Shaffer P S 1992 Research as a guide for curriculum development: an example from introductory electricity: I. Investigation of student understanding Am. J. Phys. 60 994-1003

[3] Shaffer P S and McDermott L C 1992 Research as a guide for curriculum development: an example from introductory electricity: II. Design of instructional strategies Am. J. Phys. 60 1003-13

[4] Periago M C and Bohigas X 2005 The persistence of prior concepts about electric potential, current intensity and Ohm's Law in students of engineering Revista Electrónica de Investigación Educativa 7 1-24

[5] Barbieri S 2014 Superconductivity explained with the tools of the classical electromagnetism $P h D$ Thesis Universitá degli Studi di Palermo-Dottorato di ricerca in 'Storia e Didattica delle Matematiche della Fisica e della Chimica' https://iris.unipa.it/retrieve/handle/10447/97514/ 100550/Tesi\%20Barbieri\%20Superconduttivit\%C3\%A0.pdf

[6] Sherwood B A and Chabay R W 1999 A unified treatment of electrostatics and circuits <http:// matterandinteractions.org/wp-content/uploads/2016/07/circuit.pdf>

[7] Eylon B and Ganiel U 1990 Macro-micro relationship: the missing link between electrostatics and electrodynamics in students' reasoning Int. J. Sci. Educ. 12 79-94

[8] Borges A T 1999 Mental models of electricity Int. J. Sci. Educ. 21 95-117

[9] Enghelhardt P V and Beichner R J 2004 Students' understanding of direct current resistive electrical circuits Am. J. Phys. 72 98-115

[10] John I and Allie S 2017 DC circuits: I. Evidence for fine grained contextual dependence Eur. J. Phys. 38015701 (22pp)

[11] John I and Allie S 2017 DC circuits: II. Identification of foothold ideas in DC circuits Eur. J. Phys. 38015702 (20pp)

[12] Singh C 2006 Student understanding of symmetry and Gauss's law of electricity Am. J. Phys. 74 923-36

[13] Chabay R W and Sherwood B A 2015 Matter and Interactions 4th edn (New York: Wiley)

[14] Chabay R W and Sherwood B A 2006 Restructuring the introductory electricity and magnetism course Am. J. Phys. 74 329-36

[15] Jefimenko O D 1962 Demonstration of the electric fields of current-carrying conductors Am. J. Phys. 30 19-21 
[16] tinyurl.com/SurfaceCharge

[17] Garzón I, De Cock M, Zuza K, van Kampen P and Guisasola J 2014 Probing university students' understanding of electromotive force in electricity Am. J. Phys. 82 72-9

[18] Zuza K, De Cock M, van Kampen P, Bollen L and Guisasola J 2016 University students' understanding of the electromotive force concept in the context of electromagnetic induction Eur. J. Phys. 37065709 (13pp)

[19] Giuliani G 2010 Vector potential, electromagnetic induction and 'physical meaning' Eur. J. Phys. $31871-80$

[20] Barbieri S, Cavinato M and Giliberti M 2013 An educational path for the magnetic vector potential and its physical implications Eur. J. Phys. 34 1209-19 